Voix et Images

\title{
De l'effet " premier roman " à celui de Summerhill : cette liberté si difficile, si nécessaire...
}

\section{Pierre Hébert}

Volume 14, numéro 2 (41), hiver 1989

L’édition littéraire au Québec

URI : https://id.erudit.org/iderudit/200783ar

DOI : https://doi.org/10.7202/200783ar

Aller au sommaire du numéro

Éditeur(s)

Université du Québec à Montréal

ISSN

0318-9201 (imprimé)

1705-933X (numérique)

Découvrir la revue

\section{Citer cet article}

Hébert, P. (1989). De l'effet « premier roman » à celui de Summerhill : cette liberté si difficile, si nécessaire... Voix et Images, 14(2), 339-345.

https://doi.org/10.7202/200783ar d'utilisation que vous pouvez consulter en ligne. 


\section{Roman}

\section{De l'effet «premier roman» à celui de Summerhill: cette liberté si difficile, si nécessaire...}

\section{par Pierre Hébert, Université de Toronto}

Ce que l'on pourrait appeler le phénomène «premier roman» recouvre des expériences diverses, mais aussi fort intéressantes. Tantôt, le premier roman est celui dont l'auteur ne se libère jamais, et qui sert de paramètre pour toute son œuvre. Je pense ici à l'exaspération de Savard, Menaud, Menaud toujours. Comme si je n' avais rien écrit d' autre. ${ }^{1}$ Pour combien de lecteurs Gabrielle Roy ou Ringuet ne seront-ils pas toujours les auteurs de Bonheur d'occasion ou de Trente Arpents? En revanche, Kamouraska, le Canard de bois, le Matou portent ombrage, c'est le moins qu'on puisse dire, aux œuvres antérieures d'Anne Hébert, de Louis Caron et d'Yves Beauchemin.

Je propose donc un parcours à vitesse variable de quelques «premiers romans» parus depuis un $a^{2}$. Car, ne manquons pas de le noter, les premiers 
romans ont souvent été ces derniers temps des œuvres étonnantes, chacune dans son genre, et l'on a salué avec enthousiasme, depuis 1984, le Souffle de l'Harmattan de Sylvain Trudel, les Demoiselles de Numidie de Marie José Thériault, la Note de passage de François Gravel. Le même constat favorable convient également à cette dernièrc année, et plusieurs lecteurs ont sans doute eu le plaisir de lire, par exemple, Rue dés Petits-Dortoirs ${ }^{3}$, Tout l'été dans une cabane à bateau ${ }^{4}$, la Nouvelle Alliance ${ }^{5}$, peut-être aussi la Rencontre ${ }^{6}$. Mais je retiendrai ici trois romans particulièrement significatifs de ce que j'appcllerais la recherche d'une vraie Renaissance.

\section{Le salut par l'humain}

Puisqu'il s'agit ici de premiers romans, je voudrais dire quelques mots sur une œuvre parue à la fin de l'année 1987, le Père de Lisa ${ }^{7}$, de José Fréchette. Ce court récit, livré sur le ton de la confidence, profite amplement d'un ton suffisamment dégagé, humoristique, pour éviter de tomber dans le faux sérieux, alors que le sujet eût pu s'y prêter. La narratrice, Isabelle, raconte avec le recul une expérience intérieure, ou plutôt, devrait-on dire, un moment intérieur de sa vie où elle a touché un point de bien-être, d'équilibre rares. Tout a commencé le jour où, accidentellement, elle a rencontré cette jeune fille d'une dizaine d'années, Lisa. Cettc rencontre fascine littéralement Isabelle, car Lisa représente une sorte de perfection aux confins de l'humain. Qu'à cela ne tienne: le père de Lisa, un peu plus tard, émettra le même magnétisme qui engendrera confidence, sécurité humaine et amour d'une nuit.

C'est en fait le choc de deux mondes qui est raconté ici, mais sans esprit tragique. La narratrice vivait dans un monde humain, trop humain mĉme, c'est-àdire imparfait, sans solidité. Arrivent Lisa et son père, qui font figure de phare pour une vie qui se cherche, une excellente borne routière (p. 28). C'est encore une fois un renouveau, une nouvelle naissance qui se produit ici à la suite de cette rencontre: $J^{\prime}$ avais eu à cet instant la merveilleuse impression que les choses re commençaient pour moi. Je ne sais pas. Comme si le monde m'appartenait. To ut ça parce que pendant trois minutes j'avais été plus belle que moi-même (p. 61).

La renaissance, qui est ici un véritable sentiment d'être au monde, d'appartenir à cette terre humaine, ne tire pas son origine d'une solution sociale ou collective. La libération de soi se joue sur un plan totalement individuel, contrairement en cela des cuvres qui suivent, de Christian Mistral et de Raymond Baudet.

\section{Beatnik, Hippie, Punk, Vamp...}

C'est bien vrai que, sur le plan écologique, ce monde ressemble de plus en plus à un vrai dépotoir maquillé; que, sur le plan social, les riches sont de plus en plus richcs, et les pauvres encore plus pauvres. Que, aussi, le court terme règne partout, chacun voulant sauver sa peau à défaut d'avoir pu changer la société. À défaut de rêver le monde, faisons-le au moins fonctionner convenablement.

La spirale croissante production-consommation doit recourir à la création de besoins aberrants pour se maintenir, et le matérialisme est notre peste noire. 
Et, de cela, des plus jeunes en ont marre, eux qui ont une conscience des choses trop aiguë. Ce monde, tel qu'il est, peut rendre heureux, et une Renaissance, une vraie, radicale, terrible, s'impose: voilà ce que quelques premiers romans nous donnent à lire.

Le problème du gîte réglé, comme toujours à la dernière minute, il ne nous restait plus qu'à nous enivrer de paroles (p. 99). Cette phrase suffirait presque à résumer ce roman percutant, mais, aussi, fort troublant, de Christian Mistral, Vamp ${ }^{8}$. Car le roman, à certains égards, y est contenu tout entier: ces Vamps n'ont qu'un seul besoin social (et encore!), se loger; une fois cette nécessité comblée, il ne leur reste rien à faire, sauf parler et, pour certains, écrire. Passionnant? Oui, Vamp est passionnant, mais sa vraie vie est ailleurs.

Sur le seul plan narratif, Vamp est donc fort ténu: le narrateur (l'auteur?), Christian Mistral, porte-parole de la génération Vamp, qui vit avec des semblables et en particulier avec son idole Blue Jean, est sur le point de déménager. Le roman fait état de cette migration mais, surtout, il livre les errances, les interrogations du personnage principal; et ces derniers mots sont bien usés pour tenter de décrire là où se joue l'essentiel du texte, c'est-à-dire l'image d'une génération vue à travers un témoin impitoyable. C'est ce qu'il faut examiner de plus près.

La génération Vamp est le produit ultime d'une Amérique qui tourne et tourne sur elle-même, pourrit au soleil, grouille de mouches à viande (p. 159); la génćration Vamp n'a rien à faire de ce monstre qui s'est créé ses propres besoins, sa propre suffisance, cette Amérique dans laquelle l'individu impuissant ne peut aspirer, au micux, qu'à occuper une position intéressante dans le rouage amalgamateur. Il ne subsiste dans ce monde que quelques valcurs sans effet sur le groupc, semblables à des flocons de quartz virevoltant autour du vacuum idéologique. [...] Et tout le monde s'en foutait allègrement. Nous devenions chaque jour un peu plus mous, un peu plus lâches, nous nous transformions insensiblement en chair à dictature (p. 195). Teilhard de Chardin n'avait sans doute pas prévu que le point Oméga, le pinacle évolutif (p. 76), outre qu'il est de la merde (p. 182, 207), entraine à sa suite un glissement vers le vide: Un tourbillon noir s'ouvrait sous mes pieds, un trou vide d'air et de toute autre chose, le néant cosmogonique, et c'était maintenant un lent, très lent cataclysme apocalyptique qui m'engloutissait, me précipitait moi et ma race dans l'antimatière (p. 76).

Christian Mistral et tous les autres Vamps sont pourtant des êtres humains, conscients de ce cloaque, mais aussi animés par certains rêves. Dans ce monde si peu fait pour mes désirs (p. 105), le narrateur ne peut aboutir qu'à cette évidence qu'il n'existe qu'un scul pays, le corps, le moi, et que faire à sa tête me paraissait le premier devoir de l' homme envers lui-même (p. 151). Faire à sa tĉte, ccrtes, mais pour ne rien faire, ne rien désirer, ne rien posséder, car cette tcrre n'offre rien d'obligatoire, rien de nécessaire, rien d'important (p. 340). Quand il ćcrit qu'on cst sur terre pour se soûler, Christian Mistral nous fait comprendre sa grande soif, celle d'une vie immédiate, où la quête de l'originalité à tout prix est la scule façon de se sauver. Le narrateur n'attend du tissu social ni 
sanctions ni idéals. Comme il ne changera pas le monde, il essaie d'en faire le plus possible, à défaut du mieux possible.

Dans ce roman, long cri intérieur mais aussi écriture qui radiographie notre fin de siècle, le plus déchirant est peut-être cette prise de conscience que tout nous dépasse mais que, aussi, l'esprit humain ne comprendra, ne connaîtra jamais. Albert Camus l'avait déjà exprimé dans son Mythe de Sisyphe: je ne connaîtrai jamais, je ne saurai jamais avec certitude le sens véritable de mon destin. Mais C. Mistral assume cette insuffisance de l'esprit humain jusque dans ses conséquences ultimes: je renonce à m'interroger plus avant, je vis dans le perpétuel tourment de l'incertitude. C'est la rançon inhérente à ce genre de courage (accepter qu'on ne peut savoir). Il n'y a pas d'objectivité. De plus, il n'y a de bonne subjectivité que poétique; mis en forme, le jugement partial est réhabilité sur le double plan de l'esthétique et de la morale (p. 265). C'est pourquoi la parole humaine sera désormais porteuse de vérité; mais clle la quêtera non dans la certitude des faits, non dans le sentiment d'avoir raison, mais dans une synthèse du Rien et du non-savoir. C. Mistral narrateur exprime comment écrire lui permet de se refaire une foi, d'assurer sa rédemption. Écrire est une entreprise de salut à la condition d'assumer le débordement: Voilà l'idée que je débride d'un mouvement du poignet, assis sur mon cul, j'écrase, $j$ 'écris, et c'est comme quand j' avais trois ans, qu'il fallait colorier les ballons sans en dépasser les contours: je m'en fichais, et je m'en fiche encore, je déborde, sur tout (p. 335).

Christian Mistral descend aux enfers, c'est-à-dire dans les contradictions mêmes de notre monde; il en montre les facettes les plus troublantes au moyen d'une plume en éruption, d'où coule une lave destructrice faite de mots, de hargne et de passion. Ces dernières années, on a pu sentir venir ce roman qui étalerait, là devant nous, cette Amérique qui nous tue. Le Jack Waterman de Volkswagen Blues quêtait une Amérique au visage ambigu; le Pierre de MarieClaire Blais, dans le roman du même nom, plonge tête première dans la violence américaine; pendant ce temps, les personnages de Pauline Harvey erraient dans la ville, et se droguaient à la schizophrénie. Ce jeune Mistral, je parle ici tout au moins du narrateur, qui a à peine plus de vingt ans, porte, supporte devrait-on dire, une conscience de l'inanité des agitations humaines comme s'il avait déjà vécu une vie tout entière. Il semble avoir tout vu (trop $\mathrm{vu}$ ), tout compris (trop compris), et exprime, lui, étrange animal (p. 122), combien c'était dingue, cette vie (p. 170).

\section{L'influence de la liberté ou le chercheur de trésor}

Les esthètes ne trouveront pcut-être pas leur compte dans ce roman, mais qu'importe? J'en parle ici parce que, en dépit de quelques invraisemblances, Passeport pour la liberté 9 s'inscrit dans la classe des récits conçus d'abord et avant tout pour intéresser le lecteur par son intrigue de facture traditionnelle et sa narration dirccte et efficace. Ici, et contrairement à Vamp, aucune hautevoltige stylistique: le personnage principal, François Auclair, sous-ministre associé, menait une vie confortable quand certaines exigences personnelles l'ont amené à tout plaquer là. Il prend conscience que la vie est infiniment plus riche 
que le parcours objectif que veut lui imposer la société: Le mot carrière a toujours évoqué pour moi un grand trou où l'on s'enfonce de plus en plus profondément (p. 17).

S'achetant d'abord une moto pour partir à l'aventure, puis une terre pour retrouver les bienfaits de la solitude, F. Auclair se rend compte qu'il s'est jeté dans un piège. Cette terre, dite $d u$ pendu, appartenait jadis à un certain Oram Turmel, qui, avant de mourir, y aurait enfoui quelque part un trésor, que son fils Tonio devait trouver en cinq ans, s'il continuait à travailler le sol avec persévérance. F. Auclair aura de la peine à débrouiller cette intrigue mystérieuse: car Tonio s'est pendu, et son fils Denis, duquel Auclair a acheté la terre, a perdu l'usage de ses jambes, toujours sur ce même lopin, lors de circonstances assez floues.

Centré d'abord sur cette quête du trésor, ce récit s'ouvre progressivement sur une série d'embranchements aussi multiples qu'imprévus. Car qui, à partir de cette mise en situation initiale, pourrait deviner que pareille intrigue, à travers des réflexions sur Nietzsche et saint François d'Assise, nous conduirait jusqu'en Europe?

C'est qu'en vérité, nous sommes en présence ici d'un curieux récit. De prime abord, rien que de traditionnel: la manière est directe, le style fuit les enluminures. Mais si on prend un certain recul, on se rend compte que ce qui intéresse tout autant dans Passeport pour la liberté, c'est le discours même du narrateur qui cherche à se libérer des chaînes que lui ont tendues la société, la carric̀re. Une forte exigence de libération pousse F. Auclair à mettre en relief, et parfois de manière troublante, les contradictions de notre monde et de son inlassable montée de production-consommation.

Ce roman oppose, en fait, le matérialisme et le spiritualisme de manière incessante. Tout le récit s'inscrit, à première vue, sous le signe de l'or: que celuici soit rejeté par un personnage qui en avait trop mais qui ne résista pas à la quête du trésor, par d'autres qui n'en ont pas assez et qui sont prêts à tout, le signe du dollar est omniprésent. François Auclair est attiré par la liberté intérieure de celui qui ne possède rien; mais en dernier lieu, c'est l'or qui l'emporte.

\section{Enfin, l'effet Summerhill}

Les trois romans que nous venons d'observer proposent rien de moins qu'une dissection in vitro de notre monde. Toujours dans ce même laboratoire, mais quittant l'effet "premier roman», voyons maintenant l'Effet Summerhill ${ }^{10}$, la troisième œuvre de François Gravel.

Cela se passait à une époque où une nuée d'intellectuels s'était abattue soudainement sur tout le pays (p. 11). C'est bien là le cadre de l'Effet Summerhill, mais l'on scrait tenté d'ajouter: ce roman arrive à une époque où une nuće de professeurs s'abattait soudaincment sur tout le roman québécois. Car innombrables sont les personnages-professeurs (je ne parle même pas des auteurs!) qui imposent leur présence: Maryse, l'Été Rébecca, Une histoire américaine, Agonie, Lucie ou un midi en novembre, la Note de passage, tous ces textes - et combien d'autres? - ont quelque rapport avec le merveilleux monde du ministère de 
l'Éducation. Avec l'Effet Summerhill, non seulement le personnage, mais l'histoire tout entière s'articule autour de la problématique de l'enseignement.

L'expérience éducative de Summerhill, on se le rappellera, remonte au début des annćes 20, dans la région de Londres. Il s'agissait d'une école autogérée, libre et au service de l'enfant. Dans cette école, où faire apprendre était un contresens, les valeurs sociales apparaissaient souvent comme l'inversion d'une axiologie refoulée: Le prude est un libertin qui n'a pas le courage de mettre son âme à nu, a déjà écrit A.S. Neill.

Ce qu'il faut dire, c'est que l'expérience Summerhill, qu'on y croie ou non, a entraîné une révision fondamentale de nos valeurs éducatives. Et c'est précisément en jouant sur toutes ces valeurs pour et contre Summerhill, que François Gravel s'amuse, littéralement, à projeter son lecteur dans les contradictions qu'engendrent des positions extrêmes. Le personnage principal, Jacques, a eu un père élevé à la Summerhill, une école anglaise dont il [a] toujours gardé un très mauvais souvenir... (p. 37). En conséquence, le père éduquera son fils selon une pédagogie rigoureuse et autoritaire. A-t-on deviné que le fils à son tour se rebellera et s'inscrira dans une filiale de Summerhill? Que, selon le principe d'autorcproduction de la race, ce même fils deviendra lui-même enseignant, cherchant la vérité entre Neill et son père?

François Gravcl est un écrivain «social»: il aime fréquenter les paradoxes de notre temps. Le personnage de son premier roman, la Note de passage, ouvrait le texte avec une charge contre les plus de quarante ans pour, à la fin, souhaiter entrer dans la «normalité» de ceux-là mêmes qu'il avait balayés du revers de la main. Dans l'Effet Summerhill, les mêmes balancements antinomiques circulent partout, chaque thèse, de la liberté ou de l'autorité, pouvant être retournće à l'envers comme un gant. On n'en sort jamais: Louis, le père de Jacques, aurait résisté à la liberté parce qu'elle était imposée par Neill (p. 104), et son fils se fera accuser d'abuser de [son] autorité dans le but d'imposer un certain laxisme (p. 152). Un pcu plus tard, et dans une autre école, il prononcera son serment de professeur: je multiplierai plutôt les interdits, les règlements arbitraires, les principes inutiles, ne laissant à mes élèves pour toute porte de sortie que la résistance et le rêve (p. 199).

On voit bicn comment François Gravel nous traite: son ironie ne nous laisse aucun repos. Certes les personnages parlent beaucoup dans ce roman et sans aller jusqu'à la ratiocination, lcurs visécs auraient gagné à être montrécs par le récit plutôt que dites par le discours. N'empêche que l'Effet Summerhill intéressera bien des lecteurs; non pas parce qu'il s'agit, comme on l'indique en page couverture de dos, d'une éducation sentimentale, mais bien d'une éducation à la liberté qui n'accepte, pour seule définition, que son nom même.

1 Journal manuscrit, 9 juin 1968.

2 Dois-je dire que j'ai écrit en novembre 1988 ?

3 Denis Bélanger, Rue des Petits-Dortoirs, Montréal, Québec/Amérique, 1988, 142 p. (Littérature d'Amérique). 


\section{CHRONIQUES 345}

4. Pierre Gobeil, Tout l'été dans une cabane à bateau, Montréal, Québec/Amérique, 1988, 148 p. (Littérature d'Amérique).

5 Jacques Fortier, la Nouvelle Alliance, Montréal, Quinze, 1987, 138 p.

6 Robert Barberis, la Rencontre, Montréal, Éd. du Fleuve, 1988, 91 p.

7 José Fréchette, le Père de Lisa, Montréal, Quinze, 1987, 107 p.

8 Christian Mistral, Vamp, Montréal, Québec/Amérique, 1988, 345 p. (Littérature d'Amérique).

9 Raymond Baudet, Passeport pour la liberté, Montréal, Quinze, 1988, 293 p.

10 François Gravel, l'Effet Summerhill, Montréal, Boréal, 1988, 224 p. 